\title{
The Homotopy Analysis of Unsteady Hydromagnetic Flow of a Viscous Fluid with Oscillatory Suction Velocity
}

\author{
A. S. Idowu*, O. Komolafe \\ Department of Mathematics, University of Ilorin, Ilorin Nigeria.
}

\begin{abstract}
This work considered the unsteady hydromagnetic flow of an electrically conducting, incompressible, viscous fluid past an infinite vertical porous plate. The oscillatory suction velocity is normal to the plate. The uniform magnetic field influence is normal to the flow and the permeability of the medium is time dependent. The oscillatory suction velocity was defined so as to eliminate any complexity in the equations that were derived. The problem was solved using a modified technique of the Homotopy analysis method. The results obtained were discussed for various effects of material parameters on the velocity, temperature and concentration profiles.
\end{abstract}

KEYWORDS: Heat transfer, Mass transfer, Oscillatory suction velocity, Hydromagnetic flow, Vertical porous plate

[Received March 16 2015; Revised November 10 2015; Accepted December 20 2015]

\section{INTRODUCTION}

The procedure of heat and mass transfer is seen in motion caused by buoyancy in the atmosphere. It also happens that a lot of movement processes occur in industries and natural environment in which energy and mass transfer take place at the same time. Heat and mass transfer also happen in bodies of water and inside different layers of the earth body. In chemical processes, turbo machinery, water treatment power generation and aerospace technology, unsteady oscillatory free convective flow is of very high importance. Such flows happen due to unsteady motion of a boundary layer or unsteady temperature conditions.

When it comes to industrial processes such as drying, water purification, energy transfer in cooling towers, power generation and flow in a desert cooler, a combination of heat and mass transfer problems with chemical reactions are of great importance. This type of problems have received great attention from researchers in recent years. It happens that heat and mass transfer, together with chemical reaction happen simultaneously. Possible applications of this type of flow can be found in many industries. Take for instance, in the power industry,among the methods of generating electric power is one in which electrical energy is extracted directly from a moving conducting fluid.

The study of heat generation or absorption effects in moving fluids is important in view of several physical problems, such as fluids undergoing heat-emitting or heatabsorbing chemical reactions. Effective cooling of moving machine parts and electronics have become necessary as a result of the fast growth in electronic technology. Cooling of electronic equipment ranges from computers of various sizes and uses and from energy supplies to telephone switch boards. Thermal diffusion effect has been used to separate mixtures of very light gases such as hydrogen and helium.

\footnotetext{
*Corresponding author’s e-mail address: asidowu@gmail.com
}

Gases with medium molecular weights have also been separated using their abilities to conduct heat.

In the past couple of years, an intensive research effort has been devoted to problems on heat and mass transfer in view of their application to astro-physics,geo-physics, chemistry and engineering. In addition, the problem of heat and mass transfer is also encountered in chemical process industries such as polymer production and food processing. Many researchers have studied the problems on free convection and mass transfer flow of a viscous fluid through porous media. In some studies, the permeability of the porous medium was assumed to be constant while some assumed it to be time dependent.

Panda and Mohapatra (2012) studied the effects of some pertinent parameters on unsteady oscillatory flow of an incompressible, electrically conducting viscous Liquid through a porous medium past an infinite vertical porous plate with constant suction velocity. A transverse uniform magnetic field was applied. The system was assumed to be rotating. it was shown that the effects of magnetic field and rotating parameter enhanced the velocity field. It was also realized that the rotating parameter was independent of the magnetic field. In the absence of rotation, the magnetic field has no significant contribution.

Choudhary and Bandita (2013) investigated the effects of visco-elastic parameter on the hydromagnetic oscillatory porous plate in presence of heat source. It was seen that the velocity field was significantly affected at every point of the fluid region by the visco-elastic parameter.Rao, et al (2011) studied the effects of pertinent parameters on heat and mass transfer in magneto Hydrodynamic flow of a viscous fluid past a vertical plate under oscillatory suction velocity using the finite element solution method. It was observed that velocity decreases with an increase in Hartmann number $M$ as well as Prandtl number $\mathrm{Pr}$ and Schmidt number $\mathrm{Pr}$ for doi: http://dx.doi.org/10.4314/njtd.v12i2.3 
cooling of plate $(G r>0)$, whereas velocity increases with the increase in Prandtl number $\mathrm{Pr}$ and Schmidt number $S c$ for heating of the plate $(G r<0)$. It was as well discovered that the velocity increases with an increase in permeability parameter $K_{\circ}$ for cooling of the plate $(G r>0)$ and the velocity decreases with an increase in permeability parameter $K$ 。 for heating of the plate $(G r<0)$. Furthermore, the velocity increases with an increase in Grashoff number $\mathrm{Gr}$ and modified Grashoff number $\mathrm{Gm}$. Also the, temperature and concentration decreases with an increase in Prandtl number $\operatorname{Pr}$ and Schmidt number $S c$ respectively.

Ramana (2012) investigated the effect of material parameters on MHD oscillatory flow past a vertical porous plate embedded in a rotating porous medium. It was discovered that increase in the magnetic parameter and rotation parameter has an increasing effect on the velocity profile. Decrease in permeability parameter brings about a decrease in the velocity field. The same was true of Schmidt number, the temperature drops out significantly.

Das(2010) analyzed the theoretical unsteady hydromagnetic free convective flow of a viscous incompressible electrically conducting fluid past an infinite vertical porous plate through a porous medium in the present of constant suction and heat source. The result reveals that the effect of increasing magnetic parameter $M$ retard the velocity of the flow field at all point, while an increase in Grashoff number for heat transfer or heat source/sink parameter accelerate the velocity of the flow field at all point. It also happened that for smaller values of permeability parameter, the velocity increases at all point of the flow field with increasing permeability, whereas for higher values of permeability, the effect reverses. A growing magnetic parameter $\mathrm{M}$ or Prandtl number $\mathrm{Pr}$ decelerates the temperature of the flow field at all point. While a growing permeability parameter or heat source parameter reverses the effect.

Naik (2014) studied the effect of thermal radiation on unsteady hydromagnetic free convective oscillatory couette flow of a viscous fluid embedded in a porous medium. The problem was solved using the finite difference scheme. It was observed that Grashoff and Eckert numbers tends to retard fluid velocity, whereas Hartmann number, Prandtl number, Porosity number and thermal radiation parameter have reverse effect on it. Eckert number tends to enhance fluid temperature whereas Prandtl number and Thermal radiation parameter have reverse effect on it. The profile for skin friction due to velocity profile rises under the effect of Grashoff number and Eckert number and falls under the effects of Prandtl number, porosity number and Thermal radiation parameter.

Venkateswarly, et al (2013) presented a study of unsteady MHD flow of an incompressible, electrically conducting viscous fluid past an infinite vertical porous plate under oscillatory suction velocity using the Galerkin finite method. Results were discussed for Grashoff number corresponding to the cooling and heating of the plate. It was seen that increase in Grashoff, Modified Grashoff and permeability number have an increasing effect on the velocity profiles for cooling of the plate $(G r>0)$. The Schmidt, Prandtl and Magnetic numbers have decreasing effects on the velocity profile. The reverse of these effects is the case for heating of the plate $(G r<0)$.

In previous work involving suction velocity oscillatory about a mean velocity $v_{0}$ the suction velocity is represented as $v=v_{0}\left(1+\in e^{i n t}\right)$. The problem often generate a complex part which is always discarded by researchers. Over the years, various modifications have been made to the method which was originally introduced by Liao (1991). The modification to the later is therefore referred to as the new Homotopy Analysis Method(nHAM).

It is the aim of this present work to treat the whole problem as real. The suction velocity will be represented by $v=v_{\mathrm{o}}\left(1+\in e^{n t}\right)$ where $\mathrm{n}$ is the frequency and $\mathrm{t}$ is time. This research work considered the values of y from 0 to infinity. A modified Homotopy Analysis method was used in this research work.

\section{MAterials AND Method}

An unsteady hydromagnetic flow of viscous, incompressible electrically conducting fluid pass an infinite porous plate in a porous medium of time dependent permeability and suction velocity was considered as shown in Figure 1. In Cartesian co-ordinate system, $x$ '-axis is assumed to be along the plate in the direction of the flow and y'-axis normal to it. A uniform magnetic field is introduced normal to the direction of the flow.

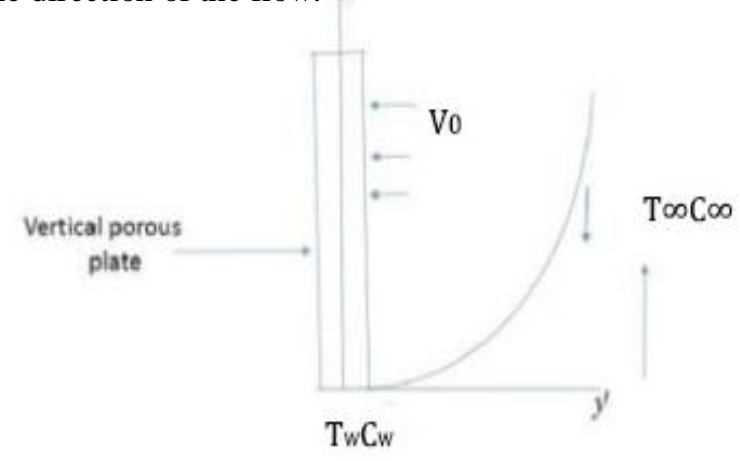

Figure 1: Physical sketch and geometry of the problem.

In the analysis, all the fluid properties are assumed to be constant except that of the influence of the density variation of the temperature. Therefore, the base flow in the medium is entirely due to buoyancy force caused by temperature and difference between the wall and medium. Initially $t^{\prime} \leq 0$, the plate as well as fluid is assumed to be at the same temperature and the concentration of species is very low. When $t^{\prime}>0$, the temperature of the plate is instataneously raised (or lowered) to $T_{w}^{\prime}$ and the concentration of the species is raised (or lowered) to $C_{w}^{\prime}$ under the stated assumption and taking the Boussinesq's approximation into account, the 
governing equations for momentum, energy and concentration in dimensionless form respectively are

Continuity Equation $\quad \frac{\partial u^{\prime}}{\partial t}=0$

$$
\begin{gathered}
\frac{\partial u^{\prime}}{\partial t^{\prime}}=g \beta\left(T^{\prime}-T_{\infty}^{\prime}\right)+g \beta^{*}\left(C^{\prime}-C_{\infty}^{\prime}\right)+\frac{v \partial^{2} u^{\prime}}{\partial y^{\prime 2}} \\
-v \frac{u^{\prime}}{k^{\prime}}-\frac{\sigma B_{\circ}^{2} u^{\prime}}{\rho} \\
\frac{\partial T^{\prime}}{\partial t^{\prime}}+v^{\prime} \frac{\partial T^{\prime}}{\partial y^{\prime}}=\frac{k}{\rho c_{p}} \frac{\partial^{2} T^{\prime}}{\partial y^{2}} \\
\frac{\partial C^{\prime}}{\partial t^{\prime}}+v^{\prime} \frac{\partial C^{\prime}}{\partial y}=D \frac{\partial^{2} C^{\prime}}{\partial y^{2}}
\end{gathered}
$$

Where $u^{\prime}$ is the velocity along the $x^{\prime}$-axis, $v$-the kinematic coefficient of viscosity, g-the acceleration due to gravity, $\beta$-the coefficient of volume expansion for the heat transfer, $\beta^{*}$-the volumetric coefficient of expansion with species concentration, $\mathrm{T}^{\prime}$-the fluid temperature, $T_{\infty}^{\prime}$-the fluid temperature at infinity, $C^{\prime}$-the species concentrated, $C_{\infty}^{\prime}$-the species concentration at infinity, D-the chemical molecular diffusivity,k-the mean assorption co-efficient, $\mathrm{k}$ ' is the constant permeability of the medium, $\rho$-the density of the fluid, $\sigma$-the electrical conductivity, $B_{\circ}$-the magnetic field strength and $\mathrm{t}$ is the time. The corresponding boundary conditions are

$$
\begin{aligned}
& t^{\prime} \leq 0: u^{\prime}=o, T^{\prime}=T_{\infty}^{\prime}, C^{\prime}=C_{\infty}^{\prime} \quad \text { forally' }^{\prime} \\
& t^{\prime}>0:\left\{\begin{array}{c}
u^{\prime}=0, T^{\prime}=T_{w}^{\prime}+\varepsilon\left(T_{w}^{\prime}-T_{\infty}^{\prime}\right) e^{\eta t}, C^{\prime} \\
=C_{w}^{\prime}+\varepsilon\left(C_{w}^{\prime}-C_{\infty}^{\prime}\right) e^{\eta t} \\
u^{\prime} \rightarrow 0 \quad T^{\prime} \rightarrow T_{\infty}^{\prime} \quad C^{\prime} \\
\rightarrow C_{\infty}^{\prime} \quad \text { asy } \rightarrow \infty \text { aty }=0
\end{array}\right.
\end{aligned}
$$

From the continuity equation, it can be seen that v' is either a constant or a function of time. So assuming suction velocity to be oscillating about a non-zero constant mean, we write $v^{\prime}=-v_{0}\left(1+\in e^{\eta t}\right)$ where $v_{\circ}$ is the mean suction velocity $\eta$-frequency of oscillation and $v_{0}>0, \in<1$ are positive constants. The negative sign indicates that the suction velocity is directed towards the plate. The permeability of the medium is considered to be $k_{\circ}\left(t^{\prime}\right)$.

\section{NON DIMENSIONALIZATION}

The non dimensionless quantities introduced in these equation were defined as:

$$
\begin{aligned}
& y=\frac{v_{\circ} y^{\prime}}{v} ; \quad t=\frac{v_{0}^{2} t^{\prime}}{4 v} ; \quad n=\frac{4 v \eta}{v_{\circ}^{2}} ; \\
& u=\frac{u^{\prime}}{v_{\circ}} ; \quad T=\frac{T^{\prime}-T_{\infty}^{\prime}}{T_{w}^{\prime}-T_{\infty}^{\prime}} \\
& C=\frac{C^{\prime}-C_{\infty}^{\prime}}{C_{w}^{\prime}-C_{\infty}^{\prime}} ; \quad k=\frac{k^{\prime} v_{\circ}^{2}}{v^{2}} ; \\
& G_{r}=\frac{v g \beta^{*}\left(T_{w}^{\prime}-T_{\infty}^{\prime}\right)}{v_{\circ}^{3}}
\end{aligned}
$$

$$
\begin{aligned}
& S c=\frac{v}{D} ; \operatorname{Pr}=\frac{\mu C_{p}}{k} ; \quad M=\frac{B_{\circ}}{v_{\circ}} \sqrt{\frac{\sigma v}{\rho}} ; \\
& G m=\frac{\nu g \beta\left(C_{w}^{\prime}-C_{\infty}^{\prime}\right)}{v_{\circ}^{3}}
\end{aligned}
$$

\section{The Homotopy Analysis Method (HAM)}

Non-linear differential equations are difficult to solve, especially analytically. The homotopy analysis method (HAM) is one of the powerful analytic method to solve differential equation with a special advantage of being able to solve non-linear differential equations. HAM enjoys great freedom in choosing initial approximations and auxiliary linear operators. The HAM can guarantee the convergence of the series solution by auxiliary parameters especially the

convergence-controller parameter $\bar{h}$. The HAM was introduced first by Liao (1991). A modified method of the HAM called the new HAM was used in this work. This modification is as a result of work done by Hassan and ELTawil (2010).

This new method of the HAM improves the convergence of the series solution, eliminates the unneeded terms and reduces time consuming in the standard HAM. An approximate solution is provided by re-writing the second order differential equation in the form of two first order differential equation. The solution of these two differential equations is obtained as a power series solution. This scheme was tested by Hassan and EL-Tawil (2010) on four exact solvable differential equations. The results demonstrated reliability and efficiency of the algorithm developed.

\section{SOlution OF THE Problem}

The equation for momentum,energy and concentration in dimensionless form are

$$
\begin{aligned}
& \frac{1}{4} \frac{\partial u}{\partial t}-\left(1+\in e^{n t}\right) \frac{\partial u}{\partial y}= \\
& (G r) T+(G m) C+\frac{\partial^{2} u}{\partial y^{2}}-\frac{u}{k_{\circ}\left(1+\in e^{n t}\right)}-M^{2} u \\
& \frac{1}{4} \frac{\partial T}{\partial t}-\left(1+\in e^{n t}\right) \frac{\partial T}{\partial y}=\frac{1}{P r} \frac{\partial^{2} T}{\partial y^{2}} \\
& \frac{1}{4} \frac{\partial C}{\partial t}-\left(1+\in e^{n t}\right) \frac{\partial C}{\partial y}=\frac{1}{S c} \frac{\partial^{2} C}{\partial y^{2}}
\end{aligned}
$$

The relevant boundary condition in dimensionless form are

$$
\left.\begin{array}{c}
u=0, \quad T=1+\in e^{\eta t}, \\
\begin{array}{c}
C=1+\in e^{\eta t} \text { aty }=0 \\
u \rightarrow 0, \quad T \rightarrow 0, \quad C \rightarrow 0 \text { asy } \rightarrow \infty
\end{array}
\end{array}\right\}
$$

Re-arranging the eqns (6)-(8), 


$$
\begin{aligned}
& \frac{\partial^{2} u}{\partial y^{2}}-\frac{1}{4} \frac{\partial u}{\partial t}+\left(1+\in e^{\eta t}\right) \frac{\partial u}{\partial y}- \\
& \left(\frac{1}{k_{\circ}\left(1+\in e^{\eta t}\right)}+m^{2}\right) u+(G r) T+(G m) C \\
& u^{\prime}=v \\
& v^{\prime}=\frac{1}{4} \frac{\partial u}{\partial t}-\left(1+\in e^{\eta t}\right) \frac{\partial u}{\partial y}+ \\
& \left(\frac{1}{k_{\circ}\left(1+\in e^{\eta t}\right)}+m^{2}\right) u-(G r) T-(G m) C \\
& u_{\mathrm{o}}(y, t)=0, \quad v_{\mathrm{o}}(y, t)=\alpha \\
& L u(y, t)=\frac{\partial u(y, t)}{\partial y} \quad L v(y, t)=\frac{\partial v(y, t)}{\partial y} \\
& A u_{m-1}(y, t)=\frac{1}{4} \frac{\partial u}{\partial t}-\left(1+\in e^{\eta t}\right) \frac{\partial u}{\partial y}+ \\
& \left(\frac{1}{k_{\circ}\left(1+\in e^{\eta t}\right)}+m^{2}\right) u-(G r) T-(G m) C \\
& B v_{m-1}(x, t)=0 \\
& u_{1}=\bar{h} \int_{0}^{y}\left(-v_{\circ}\right) d y \\
& v_{1}=\bar{h} \int_{0}^{y}\left(\begin{array}{l}
-\frac{1}{4} \frac{\partial u_{\circ}}{\partial t}+\left(1+\in e^{\eta t}\right) \frac{\partial u_{\circ}}{\partial y}- \\
\left(\frac{1}{k_{\circ}\left(1+\in e^{\eta t}\right)}+m^{2}\right) u_{\circ}+(G r) T-(G m) C
\end{array}\right) d y \\
& \text { for } m \geq 2 \\
& u_{m}(y, t)=(1+\bar{h}) u_{m-1}(x, t)+\bar{h} \int_{0}^{y}\left(-v_{m-1}(y, t)\right) d y \\
& v_{m}(y, t)=(1+\bar{h}) v_{m-1}(y, t)+ \\
& \bar{h} \int_{0}^{y}\left\{\frac{1}{4} \frac{\partial u_{m-1}}{\partial t}+\left(1+\in e^{\eta t}\right) \frac{\partial u_{m-1}}{\partial y}-\left(\frac{1}{k_{\circ}\left(1+\in e^{\eta t}\right)}+m^{2}\right) u_{\text {。 }}\right. \\
& +(G r) T-(G m) C\} d y
\end{aligned}
$$

The same procedure was followed for the energy and concentration equations with the introduction of $\beta, \gamma, \bar{h}_{1}$, and $\bar{h}_{2}$ .The values of $\alpha, \beta$ and $\gamma$ were determined by using the boundary conditions as $\mathrm{y}$ tends to infinity. The values of $\bar{h}, \bar{h}_{1}$, and $\bar{h}_{2}$ are also determined.

The mathematical software, MAPLE 13, was used to solve the system of equation up till the seventh iteration for velocity, temperature and concentration.

$$
u=\sum_{m=0}^{7} u_{m}, \quad T=\sum_{m=0}^{7} T_{m}, \quad C=\sum_{m=0}^{7} C_{m}
$$

The folowing values, $\in=0.005, n=0.5, t=3.142$ were used to determine the values of $\alpha, \beta, \gamma, \bar{h}, \bar{h}_{1}, \bar{h}_{2}$

$$
\begin{aligned}
& \alpha=20, \quad \beta=0.125, \quad \gamma=0.125, \\
& \bar{h}=-0.3, \quad \bar{h}_{1}=-1, \bar{h}_{2}=-1 .
\end{aligned}
$$

The value of $G m, G r, S c, P r, m, k$ 。 were also substituted to make the velocity,temperature and concentration equations functions of y above.

\section{Discussion Of Results}

The temperature and the species concentration were coupled to the velocity via Grashoff number (Gr) and modified Grashoff number(Gm). Figures 2-11 display the effects of material parameters $\mathrm{Gr}, \mathrm{Gm}, \mathrm{M}, \mathrm{Sc}, \mathrm{Pr}$ and $\mathrm{k}$ on the velocity, temperature and concentration fields.

For various values of Grashoff number and modified Grashoff number, the velocity profiles are plotted in Figures 2 and 3. The Grashoff number ( $\mathrm{Gr}$ ) signifies the relative effect of the thermal buoyancy force to the viscous hydrodynamics force in the boundary layer. It was observed that there was an increase in velocity field with decrease in Grashofff number.Same apply also to the modified Grashoff number. The effect of magnetic parameter $\mathrm{M}$ is shown in Figure 4.

It was observed that the velocity increases with increase in magnetic parameter $\mathrm{M}$. It is also observed that velocity increases with decrease in permeability parameter K. For the negative values of Grashoff number $(G r<0)$ i.e when the plate is being heated, Figures 8-11 shows the effects of material parameters on the velocity profile. It is seen that increase in magnetic number increases the velocity. Increase in permeability parameter number also brings about a decrease in velocity profile. Increase in Prandtl number also brings about an increase in velocity profile. Increase in Grashoff number brings about increase in the velocity profile. Increase in modified Grashoff number $\mathrm{Gm}$ increases the velocity profile.

The Prandtl number is the only parameter associated with the temperature field in the problem investigated. Figure 6 illustrates the effect of Prandtl number on the temperature profile. An increase in Prandtl number decreases the temperature field.

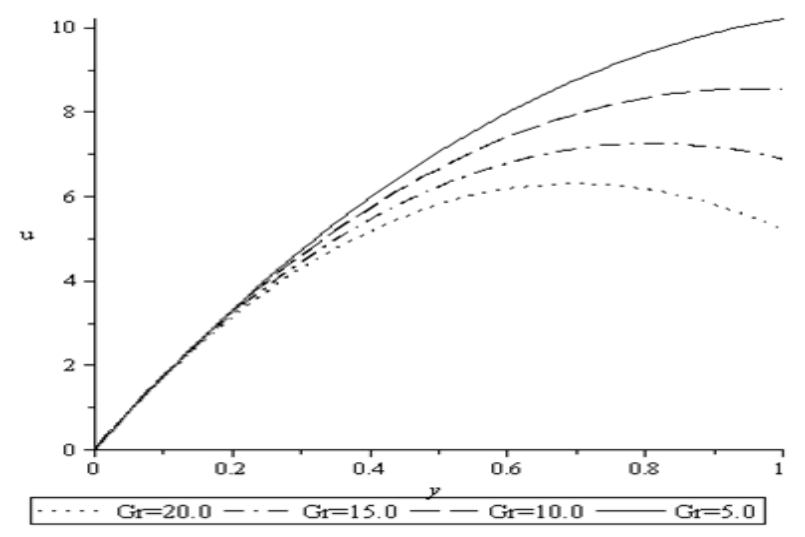

Figure 2: Effects of Grashoff numbers on velocity profile

The Schmidt number is the only parameter associated with the concentration field of the problem investigated. Figure 7 illustrate the effect of Schmidt number on the concentration profile. It is seen that an increase in Schmidt number decreases the concentration field. Also concentration field falls slowly and steadily for Hydrogen and Helium but falls rapidly for Oxygen and Ammonia in comparison to water vapour. This water vapour can be used for maintaining normal concentration field and Hydrogen can be used for maintaining effective concentration field. 


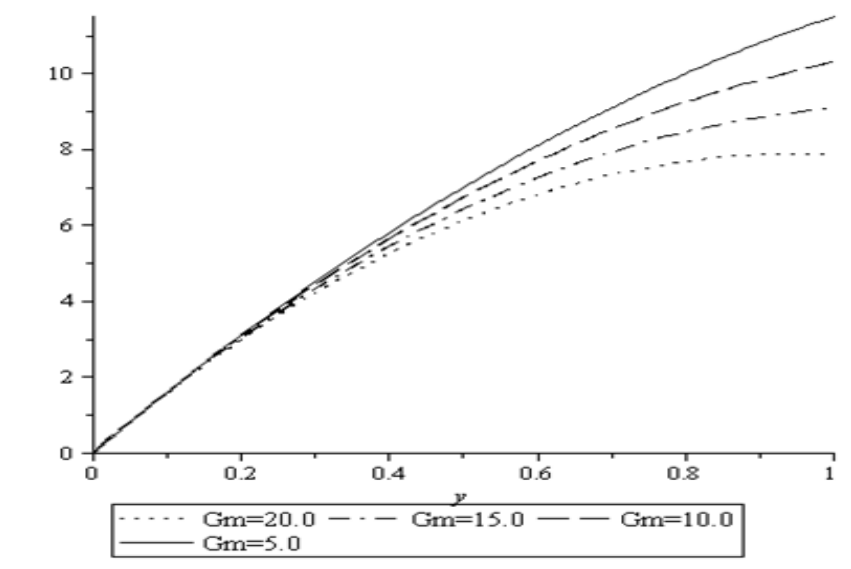

Figure 3: Effects of Modified Grashoff numbers on velocity profile

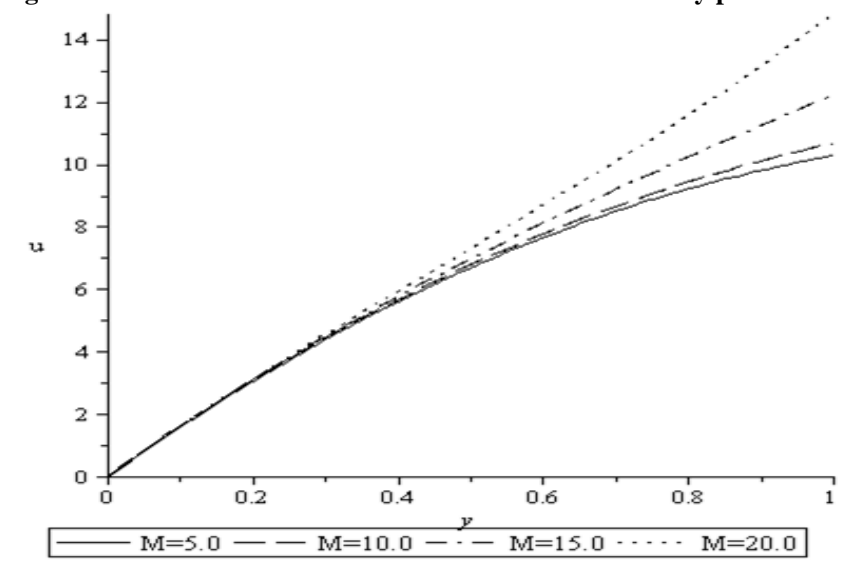

Figure 4: Effects of Magnetic number on the velocity profile
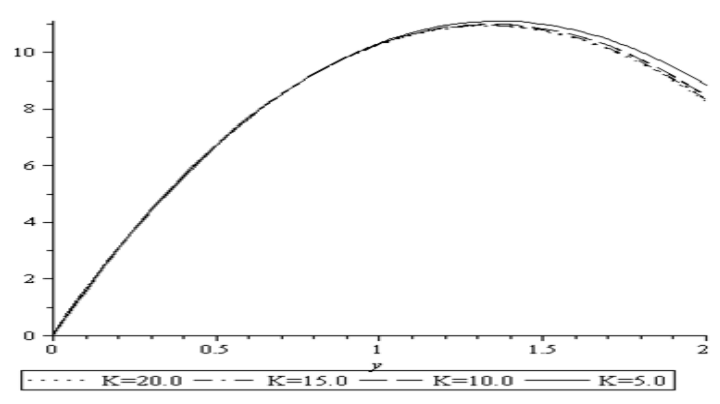

Figure 5: Effects of Permeability parameter on velocity profile

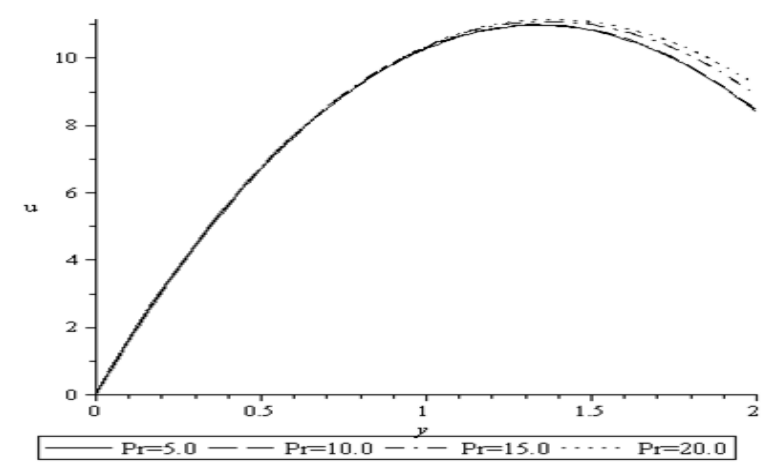

Figure 6: Effects of Prandtl number on Temperature profile

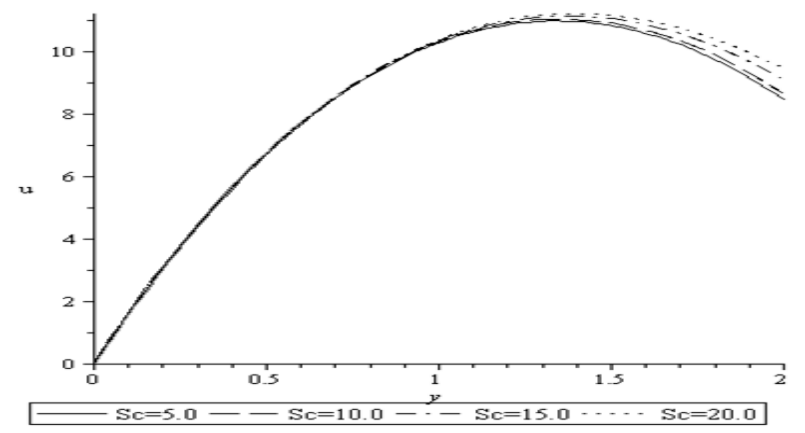

Figure 7: Effects of Schmidt number on Concentration profile

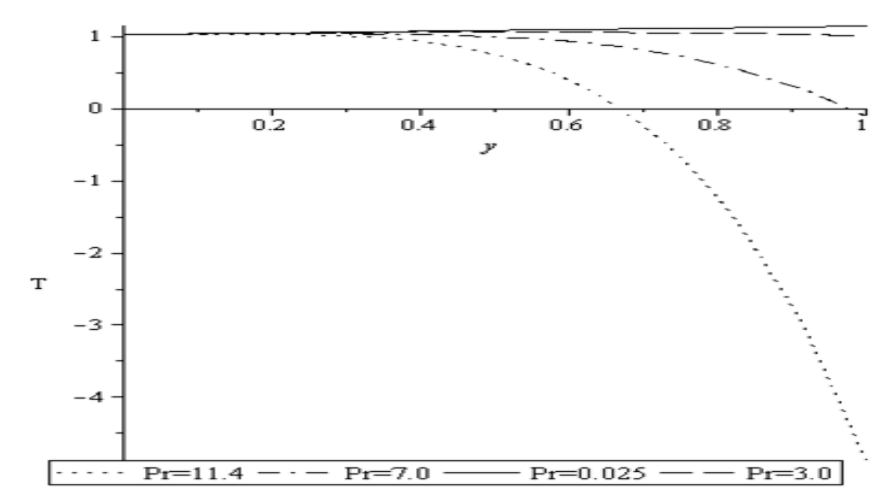

Figure 8: Effects of Grashoff number on velocity profile for ( $G r<0$ )

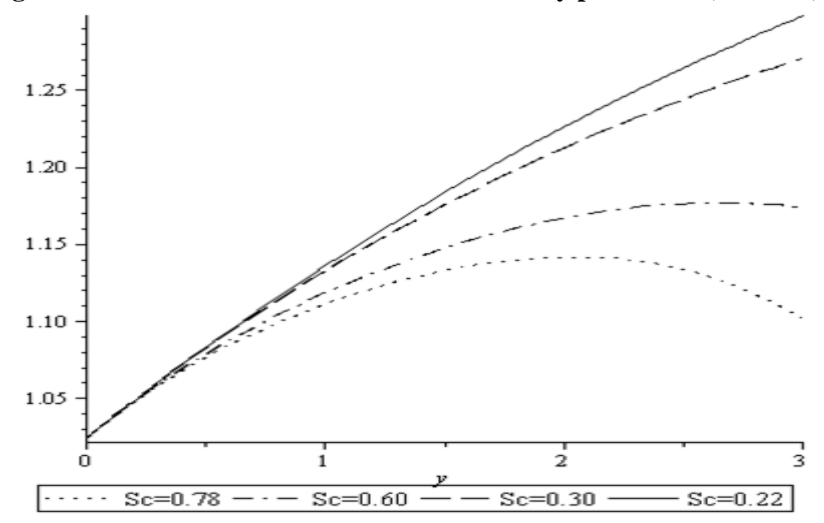

Figure 9: Effects of modified Grashoff number on velocity profile for $(\mathbf{G r}<\mathbf{0})$

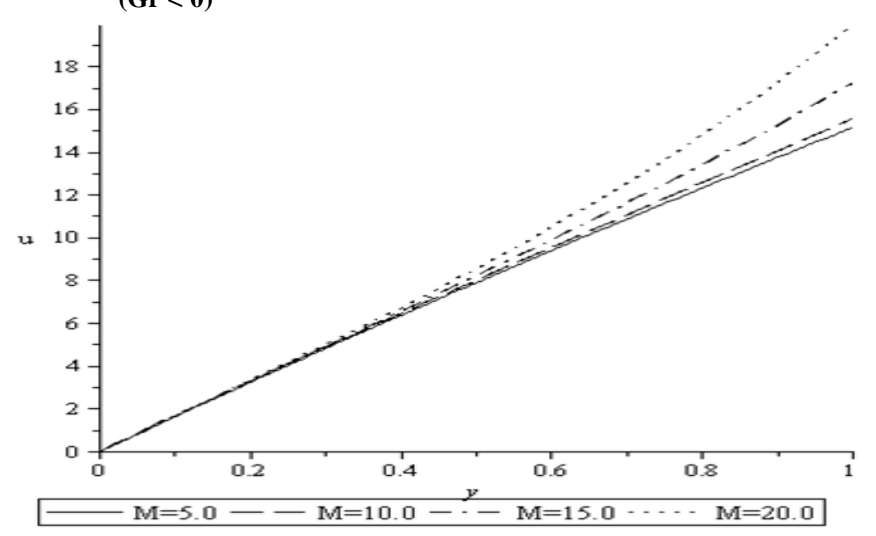

Figure 10: Effects of Magnetic number on velocity profile for $(\mathrm{Gr}<0)$ 


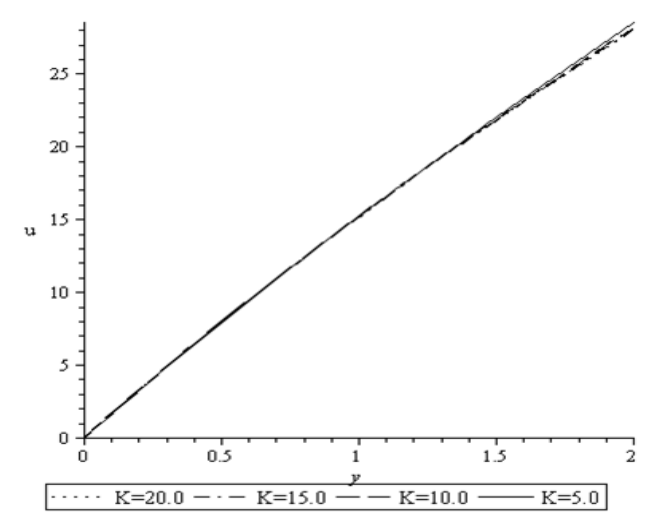

Figure 11: Effects of Permeability parameter on velocity profile for $(G r<0)$

\section{SUMMARY}

Unsteady MHD flow of an incompressible, electrical conducting, viscous fluid past an infinite vertical porous plate of time dependent permeability under oscillatory suction velocity normal to the plate has been studied. The oscillatory suction velocity was defined as $v=-v_{0}\left(1+\varepsilon e^{n t}\right)$.The arising equations namely, momentum, energy and concentration have been solved using a new technique of using Homotopy analysis method. Through this method, the velocity, temperature and concentration profiles were obtained. The effects of material parameters like the Grashoff,modified Grashoff, Prandtl, Schmidt and Hartmann numbers were checked on the velocity profile. Effect of the Prandtl number was checked on the temperature profile while varying effects of Schmidt number were checked on the concentration profile. These effects of varying parameters were shown with the help of graphs.

\section{CONCLUSION}

With the oscillatory suction velocity defined as $v^{\prime}=-v_{\text {o }}\left(1+\varepsilon e^{n t}\right)$, this took care of whatever complex expression that might have been included in the equations that were solved. The problem in this form is more applicable to real life problems. In the presence of complex expressions which would be discarded while solving, the problem would have deviated from real life applications thereby reducing the reliability of the model in relation to solving real problems. It was also observed that the new technique of using Homotopy Analysis Method proved to be more efficient, faster and easier to use in comparison to other methods.

The various effects of material parameters were checked on velocity, temperature and concentration profile. It was seen that these effects were similar to results from other researchers who defined the oscillatory suction velocity with the complex number. The similarity in results shows an efficiency in the definition of oscillatory suction velocity used for the purpose of this study.

\section{REFERENCES}

Choudhary, R., Das, B. (2013). Visco-elastic effect on MHD free convective flow past an oscillating porous plate through porous medium with heat source. International Journal of Innovative Research in science Engineering and Technology, 2: 7287-7295.

Das S. S. (2010). Hydromagnetic convective flow past a vertical porous plate through a porous medium with suction and heat source. International Journal of Energy and Environment 1(3):467-478.

Hassan, N. H., El-Tawil, M. A. (2012). A new technique of unit homotopy analysis method for solving highorder Nonlinear Differential equation. Applied Mathematics and Computation, 219: 708-728.

Komolafe, O. (2014). The Homotopy Analysis of Unsteady Hydromagnetic Flow of a Viscous Fluid with Oscillatory Suction Velocity,Unpublished M.Sc. Dissertation, 2014.

Liao, S. J. (1991). The proposed Homotopy analysis technique for the solution of no nlinear Problem, Ph.D thesis

Liao, S. J. (2003). Beyond Perturbalion: Introduction to Homotopy analysis Method, Chapman and Hall/CRL press.

Naik, S. H. (2014). Thermal radiation effect on a unsteady hydromagnetic force convective oscillatory convective flow of a viscous fluid embedded in a porous medium. IJETTCS, 3 (4): 6-12.

Panda, J. P., Mohapandra, J. K. (2012). Hydromagnetic Oscillatory flow of a viscous liquid. International Journal physics and mathematically sciences, 28: 239-243.

Ramana G. V. (2012). MHD oscillatory flow past a vertical porous plate embedded in a rotating Porous medium. International Journal of Engineering. 3: 253-258

Rao, J. A.: R. S. Raju, S. Sivaiah (2012). Finite element solution of heat and mass transfer in MHD flow of a viscous fluid past a vertical plate under oscillatory suction velocity, Journal of Applied fluid mechanics, S: 1-10

Venkateswarly, M.: G. V. Ramana Reddy, D. V. Lakshmi (2013). Unsteady under oscillatory suction velocity. Advances in Applied science Research, 4(6): 52-67. 